\title{
Approximating the Solution Stochastic Process of the Random Cauchy One-Dimensional Heat Model
}

\author{
A. Navarro-Quiles, ${ }^{1}$ J.-V. Romero, ${ }^{1}$ M.-D. Roselló, ${ }^{1}$ and M. A. Sohaly ${ }^{1,2}$ \\ ${ }^{1}$ Instituto Universitario de Matemática Multidisciplinar, Universitat Politècnica de València, Camino de Vera, \\ $s / n, 46022$ València, Spain \\ ${ }^{2}$ Department of Mathematics, Faculty of Science, Mansoura University, Mansoura, Egypt
}

Correspondence should be addressed to M.-D. Roselló; drosello@imm.upv.es

Received 9 August 2016; Accepted 27 September 2016

Academic Editor: Francisco J. S. Lozano

\begin{abstract}
Copyright (C) 2016 A. Navarro-Quiles et al. This is an open access article distributed under the Creative Commons Attribution License, which permits unrestricted use, distribution, and reproduction in any medium, provided the original work is properly cited.

This paper deals with the numerical solution of the random Cauchy one-dimensional heat model. We propose a random finite difference numerical scheme to construct numerical approximations to the solution stochastic process. We establish sufficient conditions in order to guarantee the consistency and stability of the proposed random numerical scheme. The theoretical results are illustrated by means of an example where reliable approximations of the mean and standard deviation to the solution stochastic process are given.
\end{abstract}

\section{Introduction}

The heat is the energy which flows from the higher to the lower temperature and the transport coefficient depends on the specific mode transfer. The transfer modes are the diffusive transport of thermal energy (the conduction mode), the exchange of heat between a moving fluid and an adjoining wall (the convection mode), and the radiation mode where all bodies can emit thermal radiation $[1,2]$. In a metal rod with nonuniform temperature, heat is transferred from regions of higher temperature to regions of lower temperature. Usually, the physical principles for heat transfer are heat energy of a body with uniform properties, Fourier's law of heat transfer, and conservation of energy [2-4].

When dealing with a partial differential equation together with the initial and boundary conditions, it is crucial to obtain a well-posed problem. The extent of the spatial domain is another division for the partial differential equation that makes one method of solution preferable over another. Spatial domain may be a finite interval or an infinite interval, such as the whole real line. If the spatial domain is unbounded, the boundary conditions are not an important issue and in that case the problem is called initial value problem (IVP). In mathematics, a pure IVP is usually referred to as a Cauchy problem $[3,5]$. This paper is concerned with the study of random finite difference schemes (one of the most widely used methods for engineering models) to the Cauchy problem for the one-dimensional random heat equation with unbounded spatial domain

$$
u_{t}(x, t)=\beta u_{x x}(x, t), \quad t>0,-\infty<x<\infty,
$$

with initial condition

$$
u(x, 0)=u_{0}(x) .
$$

In this IVP (1)-(2), $t$ is the time variable, $x$ is the space coordinate, $u_{t}$ and $u_{x x}$ denote the first and the second derivatives with respect to $t$ and $x$, respectively, and $\beta$ is a random variable defined in a probability space $(\Omega, \mathscr{F}, \mathbb{P})$. In addition, $u_{0}(x)$ is an initial deterministic data function. Expression (1) is a random parabolic partial differential equation for temperature $u(x, t)$ in a heat conducting insulated impurity rod along the $x$-axis since the conductivity coefficient, $\beta$, is assumed to be a random variable. The physical significance of thermal diffusion coefficient is associated with the speed of the flux of heat into the material when changes of temperature take place over the time. The heating propagation rate is proportional to the thermal diffusivity [6]. As it is stated in 
the thermodynamics' laws, $\beta$ should be a function of two independent and intensive dynamic properties (usually, temperature and pressure) [7]. From the second thermodynamics law, it is required that $\beta$ be positive. In this paper, we take $\beta$ as a random variable since the randomness of heat transfer depends on the randomness of the conductivity coefficient. The randomness of $\beta$ may be from the impurity material properties used to make the rod.

Mathematical models described by means of partial differential equations (PDEs) appear often in many areas of science and engineering and also in medicine and finance, for example, [8-10]. The heat equation has a great deal of application in many branches of sciences, naturally in a variety of models from chemistry, theoretical physics, and others [1]. The Cauchy problem model (1)-(2) is set on an unbounded space domain, so we do not need boundary conditions explicitly. There are analytical and numerical methods for dealing with problem (1)-(2) in case the conductivity diffusive coefficient is a constant or a deterministic function.

In the deterministic scenario, the heat equation on unbounded domains has been studied by different authors [11-13]. This important Cauchy problem appears in areas such as acoustic, electrodynamics, and fluid mechanics [12, 14, 15]. In the random context, the heat model has been studied using analytical techniques based on random Fourier series [16] or random Fourier integral transforms [17, 18]. In all of these contributions uncertainty is considered throughout a very general pattern and they are solved using the socalled $L_{p}$-random calculus $[19,20]$. The heat model has also been treated considering that randomness is a white noise (the derivative of the Brownian motion or Wiener process). This approach requires the so-called Itô calculus $[21,22]$. Under this approach the uncertainty is assumed to be Gaussian. This is a nice statistical property that has enabled the development of both analytic and numerical methods to study the stochastic heat model [23].

In this paper, we propose a random finite numerical scheme to approximate the solution s.p. of the Cauchy problem (1)-(2) and we prove its consistency and stability in a random sense that will be specified later. An important issue regarding our study is that we permit that, apart from the Gaussian distribution, r.v. $\beta$ that appears in the PDE (1) can also have another quite general probability distributions.

This paper is organized as follows. In Section 2 firstly a random numerical finite difference scheme for the Cauchy problem (1)-(2) is proposed. Secondly, sufficient conditions for the consistency and stability of the random numerical scheme are given. Section 3 addresses the illustration of the theoretical results established by means of an illustrative example. Conclusions are drawn in Section 4.

\section{Random Finite Difference Technique}

This section is devoted to introducing the numerical technique that will be considered later in order to approximate the solution s.p. to the random IVP (1)-(2). Firstly, it is convenient to introduce some notation that will be used throughout our analysis. With this goal, let us consider a uniform space grid $\Delta x$ and a uniform time grid $\Delta t$ which defines a two-dimensional space-time mesh grid where the exact solution s.p. to the random IVP (1)-(2), $u(x, t)$, will be approximated. This approximation at the point $\left(x_{k}, t_{n}\right)=$ ( $k \Delta \mathrm{x}, n \Delta t)$ or the mesh grid point $(k, n), k \in \mathbb{Z}, n \in \mathbb{N}$, will be denoted by $u_{k}^{n}$; that is, $u_{k}^{n} \approx u\left(x_{k}, t_{n}\right)$.

The next step is to approximate the solution s.p. to the IVP (1)-(2) on the mesh grid using some kind of approximation of the partial derivatives that appear in the formulation of that problem. In this paper, the following time-forward and spacecentered discretization will be considered:

$$
\begin{aligned}
u_{t}(k \Delta x, n \Delta x) & \approx \frac{u_{k}^{n+1}-u_{k}^{n}}{\Delta t}, \\
u_{x x}(k \Delta x, n \Delta x) & \approx \frac{u_{k+1}^{n}-2 u_{k}^{n}+u_{k-1}^{n}}{(\Delta x)^{2}} .
\end{aligned}
$$

Substituting the approximations (3) in (1)-(2), one obtains the following random finite difference scheme (RFDS):

$$
\begin{aligned}
u_{k}^{n+1} & =(1-2 r) u_{k}^{n}+r u_{k+1}^{n}+r u_{k-1}^{n}, \\
u_{k}^{0} & =u_{0}(k \Delta x),
\end{aligned}
$$

where

$$
r=\beta \frac{\Delta t}{(\Delta x)^{2}} .
$$

As it is well known from the deterministic case, the study of the consistency and stability is a main issue when dealing with numerical schemes. This motivates the analysis of consistency and stability of the random numerical scheme (4)-(5) in a stochastic sense that will be specified later. Since the approximations of the solution s.p. to the IVP (1)-(2) will be constructed in the sense of fixed station for the time, hereinafter we will work in the following Banach space $\left(\ell_{2}(\Omega),\|\cdot\|_{\mathrm{RV}}\right)[24]$ defined by

$$
\begin{aligned}
& \ell_{2}(\Omega)=\left\{\mathbf{v}=\left(\ldots, v_{-1}, v_{0}, v_{1}, \ldots\right):\|\mathbf{v}\|_{\mathrm{RV}}<+\infty\right\}, \\
& \|\mathbf{v}\|_{\mathrm{RV}}=\left(\mathbb{E}\left[\left(\sup _{k}\left|v_{k}\right|\right)^{2}\right]\right)^{1 / 2},
\end{aligned}
$$

where $\mathbb{E}[\cdot]$ denotes the expectation operator. Notice that the supremum in (7) is taken for every $k \in \mathbb{Z}$; however in order to simplify the notation, henceforth we will omit the symbol $\mathbb{Z}$.

2.1. Study of the Consistency of the Random Finite Difference Numerical Scheme. According to the definition of the consistency of a finite difference numerical scheme in the deterministic case, below we extend this definition to the random scenario taking into account the norm (7). For the sake of completeness, we also introduce the definition of the order of a RFDS as a natural generalization of the classical definition.

Definition 1. The random finite difference scheme

$$
\mathbf{u}^{n+1}=Q\left(\mathbf{u}^{n}\right)+\Delta t \mathbf{G}^{n},
$$

being

$$
\begin{aligned}
\mathbf{u}^{n} & =\left(\ldots, u_{-1}^{n}, u_{0}^{n}, u_{1}^{n}, \ldots\right)^{\top}, \\
\mathbf{G}^{n} & =\left(\ldots, G_{-1}^{n}, G_{0}^{n}, G_{1}^{n}, \ldots\right)^{\top},
\end{aligned}
$$


is said to be mean square $\|\cdot\|_{\mathrm{RV}}$ consistent with the random partial differential equation (RPDE) $\mathscr{L} u=F$, if the solution stochastic process (s.p.) of the RPDE, $u$, satisfies

$$
\begin{gathered}
\mathbf{u}^{n+1}=Q\left(\mathbf{u}^{n}\right)+\Delta t \mathbf{G}^{n}+\Delta t \boldsymbol{\tau}^{n}, \\
\left\|\boldsymbol{\tau}^{n}\right\|_{\mathrm{RV}} \underset{\Delta t \rightarrow 0}{\stackrel{\Delta x \rightarrow 0}{\longrightarrow}} 0,
\end{gathered}
$$

where the $k$ th component of $\mathbf{u}^{n}$ in (10) is

$$
u_{k}^{n}=u\left(x_{k}, t_{n}\right)
$$

Definition 2. In the context of Definition 1, the RFDS is said to be of order $(p, q)$ if

$$
\left\|\tau^{n}\right\|_{\mathrm{RV}}=\mathcal{O}\left((\Delta t)^{p}\right)+\mathcal{O}\left((\Delta x)^{q}\right) .
$$

Next, we shall prove that the RFDS (4)-(5) is mean square $\|\cdot\|_{\mathrm{RV}}$ consistent with the random IVP (1)-(2).

Proposition 3. Let us consider the random IVP (1)-(2) and assume that its solution s.p. $u(x, t)$ satisfies

$$
u_{t t}(x, t), u_{x x x x}(x, t)
$$

are uniformly bounded for every $(x, t)$,

$$
x \in \mathbb{R}, t>0 .
$$

Then, the RFDS (4)-(5) is mean square $\|\cdot\|_{R V}$ consistent. Moreover, this scheme has order $(p, q)=(1,2)$.

Proof. Let us denote $u_{k}^{n}$ as the exact value of the solution s.p. $u(x, t)$ at the mesh grid point $\left(x_{k}, t_{n}\right)$. Based on expression (10) of Definition 1 with $\mathbf{G}=\mathbf{0}$ and expressions (4) and (5), let us consider the Taylor expansion of the $k$ th component of $\mathbf{u}^{n+1}-Q\left(\mathbf{u}^{n}\right)$, bearing in mind hypotheses (14) and

$$
\begin{aligned}
& \left(\mathbf{u}^{n+1}-Q\left(\mathbf{u}^{n}\right)\right)_{k}=u_{k}^{n+1}-(1-2 r) u_{k}^{n}-r u_{k+1}^{n}-r u_{k-1}^{n} \\
& =u_{k}^{n+1}-u_{k}^{n}-r\left\{u_{k+1}^{n}-2 u_{k}^{n}+u_{k-1}^{n}\right\}=\left[u_{k}^{n}+\left(u_{t}\right)_{k}^{n}\right. \\
& \left.\cdot \Delta t+\mathcal{O}\left((\Delta t)^{2}\right)\right]-u_{k}^{n}-r\left\{\left[u_{k}^{n}+\left(u_{x}\right)_{k}^{n} \Delta x\right.\right. \\
& \left.+\left(u_{x x}\right)_{k}^{n} \frac{(\Delta x)^{2}}{2}+\left(u_{x x x}\right)_{k}^{n} \frac{(\Delta x)^{3}}{6}+\mathcal{O}\left((\Delta x)^{4}\right)\right] \\
& -2 u_{k}^{n}+\left[u_{k}^{n}-\left(u_{x}\right)_{k}^{n} \Delta x+\left(u_{x x}\right)_{k}^{n} \frac{(\Delta x)^{2}}{2}\right. \\
& \left.\left.-\left(u_{x x x}\right)_{k}^{n} \frac{(\Delta x)^{3}}{6}+\mathcal{O}\left((\Delta x)^{4}\right)\right]\right\}=\left\{\left(u_{t}\right)_{k}^{n}\right. \\
& \left.-\beta\left(u_{x x}\right)_{k}^{n}\right\} \Delta t+\mathcal{O}\left((\Delta t)^{2}\right)+\mathcal{O}\left(\Delta t(\Delta x)^{2}\right) .
\end{aligned}
$$

Since $u$ is a solution of random IVP (1)-(2), one gets $\left(u_{t}\right)_{k}^{n}-$ $\beta\left(u_{x x}\right)_{k}^{n}=0$ and hence the first term of the right-hand side of (15) vanishes. Then

$$
\begin{aligned}
\Delta t \tau_{k}^{n} & =\left(\mathbf{u}^{n+1}-\mathcal{Q}\left(\mathbf{u}^{n}\right)\right)_{k} \\
& =\mathcal{O}\left((\Delta t)^{2}\right)+\mathcal{O}\left(\Delta t(\Delta x)^{2}\right), \\
\tau_{k}^{n} & =\mathcal{O}(\Delta t)+\mathcal{O}\left((\Delta x)^{2}\right) .
\end{aligned}
$$

Now, taking into account (7) and (16) one gets

$$
\left\|\boldsymbol{\tau}^{n}\right\|_{\mathrm{RV}}=\left(\mathbb{E}\left[\left(\sup _{k}\left|\tau_{k}^{n}\right|\right)^{2}\right]\right)^{1 / 2} \underset{\Delta t \rightarrow 0}{\stackrel{\Delta x \rightarrow 0}{\longrightarrow}} 0
$$

and the order of the scheme is $(p, q)=(1,2)$.

2.2. Study of the Stability of the Random Finite Difference Numerical Scheme. Following the same idea we have used for introducing the concept of random consistency, below we extend the deterministic definition of stability of a finite numerical scheme to the random scenario using the norm (7).

Definition 4. The random finite difference scheme (8) is said to be mean square $\|\cdot\|_{\mathrm{RV}}$ stable if there exist positive constants $\epsilon, \delta>0$, and nonnegative constants $\eta$, $\xi$ such that

$$
\left\|\mathbf{u}^{n}\right\|_{\mathrm{RV}} \leq \eta e^{\xi t}\left\|\mathbf{u}^{0}\right\|_{\mathrm{RV}}
$$

for $0 \leq t \leq(n+1) \Delta t, 0<\Delta x \leq \epsilon$, and $0<\Delta t \leq \delta$.

Below, we establish conditions under which the RFDS (8) is mean square $\|\cdot\|_{\mathrm{RV}}$ stable.

Proposition 5. Let us consider the random IVP (1)-(2) where $\beta$ is a positive and bounded r.v.,

$$
0<\beta(\omega) \leq \beta_{1}, \quad \omega \in \Omega, \beta_{1} \in \mathbb{R} .
$$

Then, under the condition

$$
\Delta t \leq \frac{(\Delta x)^{2}}{2 \beta_{1}}
$$

the RFDS (4)-(5) is mean square $\|\cdot\|_{R V}$ stable.

Proof. Taking into account the definition of the norm (7), the definition of mean square $\|\cdot\|_{\mathrm{RV}}$ stability (see (18)), and the RFDS (4)-(5), let us consider

$$
\begin{aligned}
& \left(\left\|\mathbf{u}^{n+1}\right\|_{\mathrm{RV}}\right)^{2}=\mathbb{E}\left[\left(\sup _{k}\left|u_{k}^{n+1}\right|\right)^{2}\right]=\mathbb{E}\left[\sup _{k}\left|u_{k}^{n+1}\right|^{2}\right] \\
& =\mathbb{E}\left[\sup _{k}\left|(1-2 r) u_{k}^{n}+r u_{k+1}^{n}+r u_{k-1}^{n}\right|^{2}\right] \\
& \leq \mathbb{E}\left[\sup _{k}\left[\left|(1-2 r) u_{k}^{n}\right|+\left|r u_{k+1}^{n}\right|+\left|r u_{k-1}^{n}\right|\right]^{2}\right]
\end{aligned}
$$




$$
\begin{aligned}
& =\mathbb{E}\left[\operatorname { s u p } _ { k } \left[(1-2 r)^{2}\left|u_{k}^{n}\right|^{2}+r^{2}\left|u_{k+1}^{n}\right|^{2}+r^{2}\left|u_{k-1}^{n}\right|^{2}\right.\right. \\
& +2 r|1-2 r|\left|u_{k}^{n} u_{k+1}^{n}\right|+2 r|1-2 r|\left|u_{k}^{n} u_{k-1}^{n}\right| \\
& \left.\left.+2 r^{2}\left|u_{k+1}^{n} u_{k-1}^{n}\right|\right]\right]=\mathbb{E}\left[(1-2 r)^{2} \sup _{k}\left|u_{k}^{n}\right|^{2}+r^{2}\right. \\
& \cdot \sup _{k}\left|u_{k}^{n}\right|^{2}+r_{k}^{2} \sup _{k}\left|u_{k}^{n}\right|^{2}+2 r|1-2 r| \sup _{k}\left|u_{k}^{n}\right|^{2} \\
& \left.+\underset{k}{2 r}|1-2 r| \sup _{k}\left|u_{k}^{n}\right|^{2}+2 r^{2} \sup _{k}\left|u_{k}^{n}\right|^{2}\right]=\mathbb{E}[[(1 \\
& \left.-\underset{k}{2 r})^{2}+r^{2}+r^{2}+2 r|1-2 r|+2 r|1-2 r|+2 r^{2}\right] \\
& \left.\cdot \sup _{k}\left|u_{k}^{n}\right|^{2}\right]=\mathbb{E}\left[\left[(1-2 r)^{2}+4 r^{2}+4 r|1-2 r|\right]\right. \\
& \left.\cdot \sup _{k}\left|u_{k}^{n}\right|^{2}\right],
\end{aligned}
$$

where we have used that r.v. $r$ is positive because $\beta$ is a positive r.v. (see (5) and (19)).

Under hypotheses (19)-(20), we can assure that r.v. $r=$ $r(\omega)$ satisfies

$$
0 \leq r \leq \frac{1}{2}
$$

for all $\omega \in \Omega$; hence $|1-2 r|=1-2 r$. Therefore

$$
(1-2 r)^{2}+4 r^{2}+4 r|1-2 r|=1,
$$

for all $\omega \in \Omega$. Then applying recursively (21), one obtains

$$
\begin{aligned}
\left(\left\|\mathbf{u}^{n+1}\right\|_{\mathrm{RV}}\right)^{2} & \leq \mathbb{E}\left[\sup _{k}\left|u_{k}^{n}\right|^{2}\right]=\left(\left\|\mathbf{u}^{n}\right\|_{\mathrm{RV}}\right)^{2} \leq \cdots \\
& \leq\left(\left\|\mathbf{u}^{0}\right\|_{\mathrm{RV}}\right)^{2},
\end{aligned}
$$

or equivalently

$$
\left\|\mathbf{u}^{n+1}\right\|_{\mathrm{RV}} \leq\left\|\mathbf{u}^{0}\right\|_{\mathrm{RV}} .
$$

To summarize, condition (18) holds for $\eta=1$ and $\xi=0$.

Remark 6. It is important to point out that the hypothesis of boundedness on r.v. $\beta$ assumed in (19) in order to guarantee the mean square $\|\cdot\|_{\mathrm{RV}}$ stability of the RFDS (4)-(5) is not restrictive from a practical standpoint. Indeed, the classical Chebyshev inequality assures that any second-order random variable, with mean $\mu_{\beta}$ and standard deviation $\sigma_{\beta}$, can be approximated by truncating adequately its domain. Using this result it is easy to prove that the truncated interval $\left[\mu_{\beta}-10 \sigma_{\beta}, \mu_{\beta}+10 \sigma_{\beta}\right]$ contains $99 \%$ of the probability of $\beta$ regardless of the distribution of $\beta$. The larger the truncated interval, the better the probabilistic approximation. Naturally, the diameter of the truncation interval can be shortened if the probability distribution of $\beta$ is known. For example, if $\beta$ is an unbounded r.v. having a Gaussian distribution, $\beta \sim$ $N\left(\mu_{\beta} ; \sigma_{\beta}\right)$, then the truncation over the domain $\left[\mu_{\beta}-3 \sigma_{\beta}, \mu_{\beta}+\right.$ $3 \sigma_{\beta}$ ] contains $99.7 \%$ of the probability of $\beta$.

\section{Numerical Example}

This section is devoted to illustrating the theoretical results previously established by means of a test example where reliable approximations for the mean and the standard deviation (or equivalently the variance) of the solution s.p. of IVP (1)(2) are given. These approximations are constructed using the RFDS (4)-(5). These approximations are compared with the corresponding exact values since the example has been chosen in such a way that both the mean and the standard deviation of the solution s.p. are available.

Let us consider the random Cauchy problem (1)-(2) where $\beta$ is a r.v. of parameters $(a ; b)=(2 ; 3), \beta \sim \operatorname{Be}(2 ; 3)$, and the initial condition is $u_{0}(x)=\exp \left(-x^{2}\right)$. The exact solution s.p. of (1)-(2) is given by

$$
u(x, t)=\frac{\exp \left(-x^{2} /(1+4 \beta t)\right)}{\sqrt{1+4 \beta t}} .
$$

We will approximate the mean and standard deviation of the solution s.p., $u(x, t)$, of the random Cauchy problem (1)(2) on the spatial domain $-2 \leq x \leq 2$ using the RFDS (4)-(5). In order to guarantee the mean square $\|\cdot\|_{\mathrm{RV}}$ stability of this scheme, first we fix the space step $\Delta x$ and we take $\beta_{1}=1$, since $\beta \sim \operatorname{Be}(2 ; 3)$; then according to Proposition 5 (see condition (20)), the time step $\Delta t$ must be taken satisfying the following condition:

$$
\Delta t \leq \frac{(\Delta x)^{2}}{2}
$$

In order to compute approximations of the mean and the standard deviation of the solution s.p. $u(x, t)$ at the mesh grid point $\left(x_{k}, t_{n}\right)$, we will apply recursively the numerical scheme (4)-(5) and then we will take the expectation operator. The numerical results will be compared with the ones obtained from expression (26) using the following expression:

$$
\mathbb{E}[u(x, t)]=\int_{0}^{1} \frac{\exp \left(-x^{2} /(1+4 \beta t)\right)}{\sqrt{1+4 \beta t}} f_{\beta}(\beta) \mathrm{d} \beta,
$$

for the mean, and

$$
\begin{aligned}
& \sigma[u(x, t)] \\
& =\sqrt{\int_{0}^{1} \frac{\exp \left(-2 x^{2} /(1+4 \beta t)\right)}{1+4 \beta t} f_{\beta}(\beta) \mathrm{d} \beta-(\mathbb{E}[u(x, t)])^{2},}
\end{aligned}
$$

for the standard deviation, being

$$
f_{\beta}(\beta)=\frac{\Gamma(a+b)}{\Gamma(a) \Gamma(b)} \beta^{a-1}(1-\beta)^{b-1}, \quad a=2, \quad b=3 .
$$

In Figure 1 we show a comparison at the time instant $t=2 / 5$ (time fixed station) of the expectation of the exact solution s.p. and the approximations of the expectations using the random numerical scheme (4)-(5) with different spatial steps

$$
\Delta x=\frac{1}{16}, \frac{1}{8}, \frac{1}{4}
$$




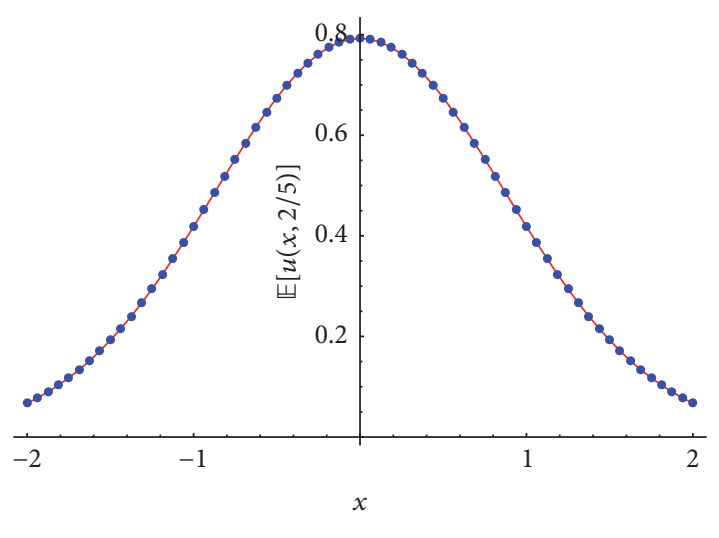

Exact

- $\Delta t=1 / 560, \Delta x=1 / 16$

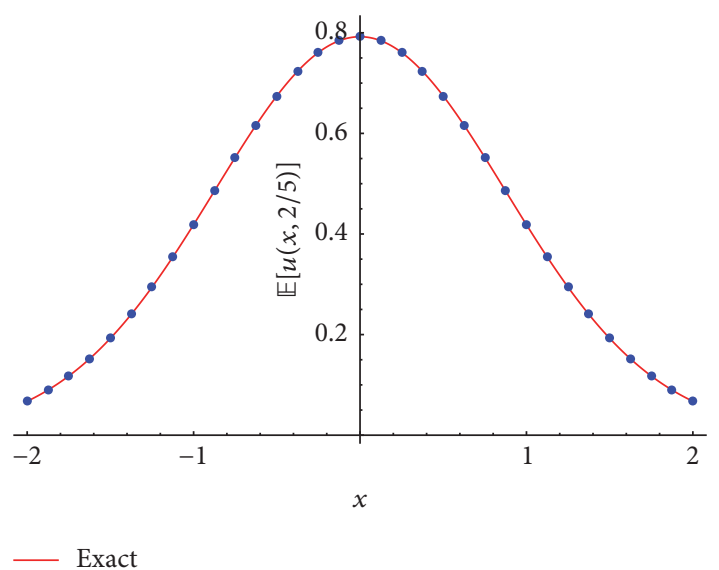

- $\Delta t=1 / 140, \Delta x=1 / 8$

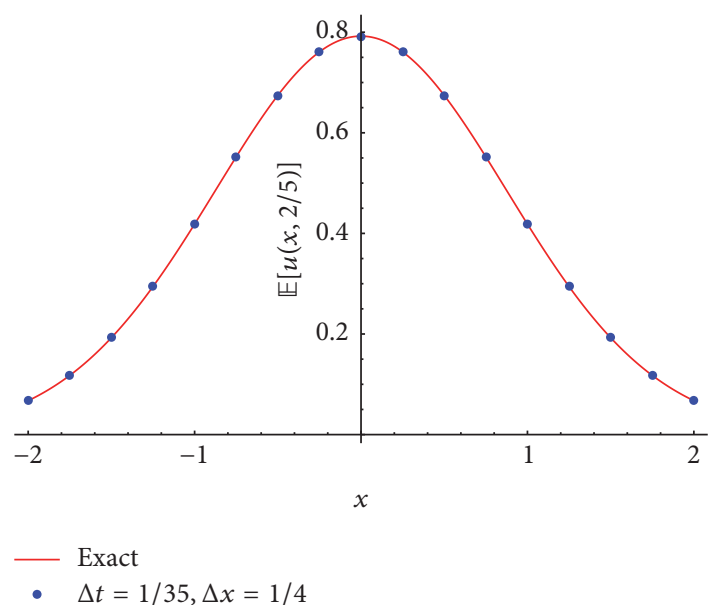

Figure 1: Expectation of the exact solution s.p. and the approximations at the time instant $t=2 / 5$ for different values of $\Delta x$ and $\Delta t$ over the spatial domain $-2 \leq x \leq 2$.

The time steps have been chosen as

$$
\Delta t=\frac{1}{560}, \frac{1}{140}, \frac{1}{35},
$$

respectively, so that stability condition (27) is guaranteed.
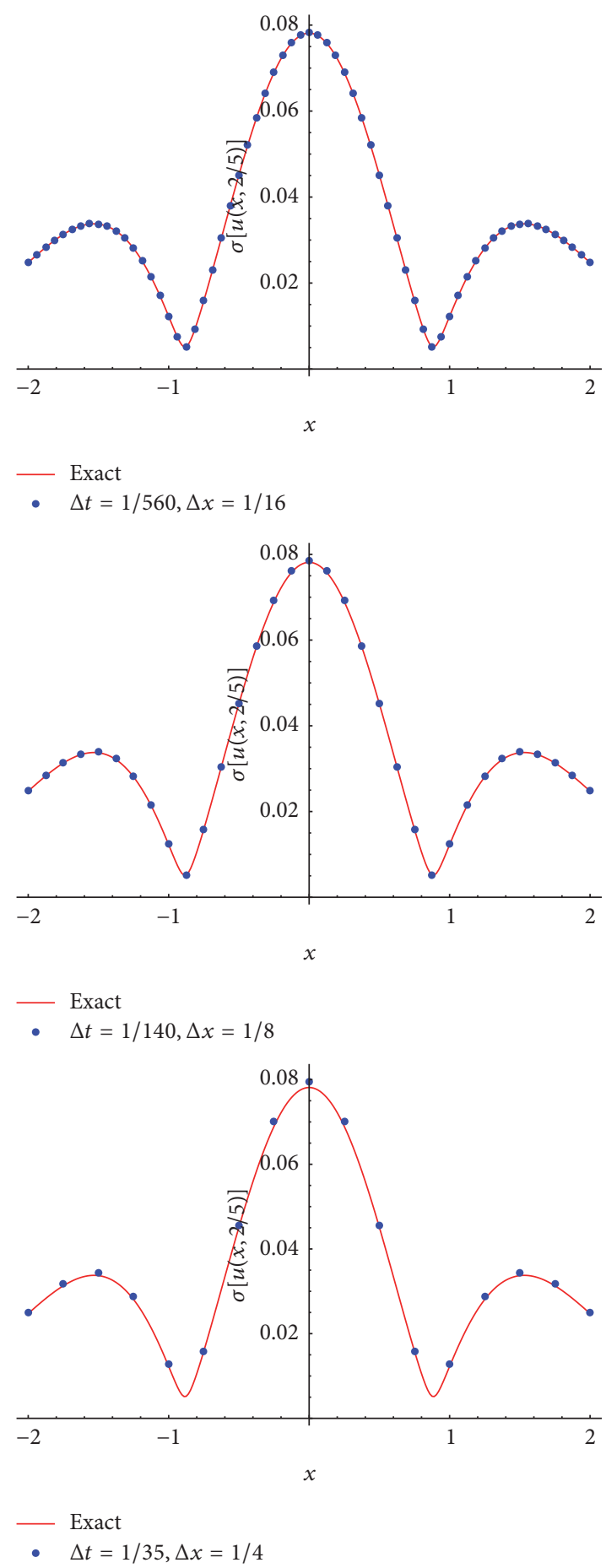

Figure 2: Standard deviation of the exact solution s.p. and the approximations at the time instant $t=2 / 5$ for different values of $\Delta x$ and $\Delta t$ over the spatial domain $-2 \leq x \leq 2$.

An analogous comparison for the standard deviation at the time instant $t=2 / 5$ is shown in Figure 2 .

To complete the numerical analysis, in Figures 3 and 4 we have plotted the relative errors for the approximations of the expectation and standard deviation for the spatial and time steps previously chosen, respectively. From these plots 

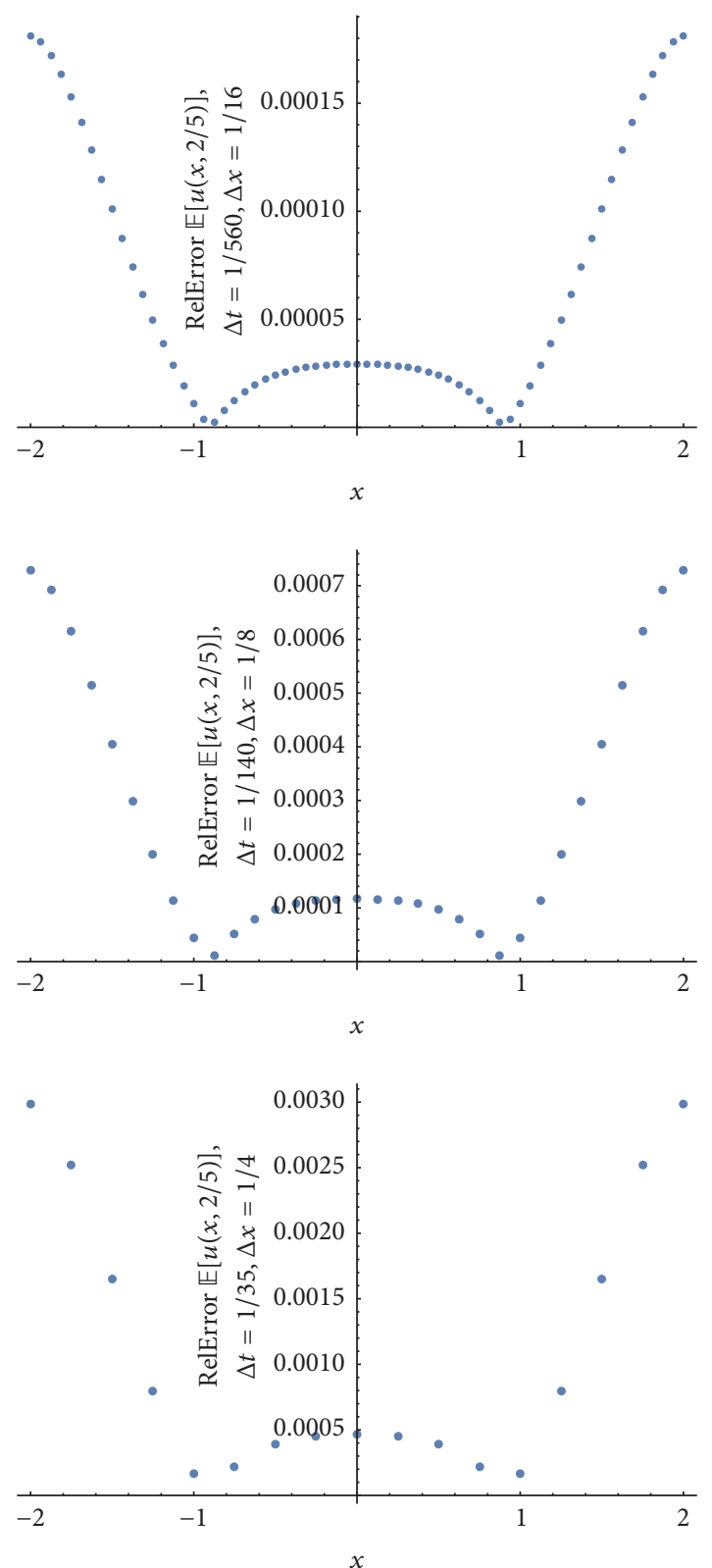

Figure 3: Relative errors at the time instant $t=2 / 5$ for the approximations of the expectation for different values of $\Delta x$ and $\Delta t$ over the spatial domain $-2 \leq x \leq 2$.

we observe that as $\Delta x$ is divided by 2 , the relative error is approximately divided by 4 . This confirms the convergence of the random numerical scheme.

\section{Conclusions}

In this paper we have studied the randomized Cauchy heat model by assuming that the diffusion coefficient is a random variable and considering a deterministic initial condition over an unbounded domain. Thus, boundary conditions have not been required. We have proposed a random finite difference scheme for solving this model. The mean square consistency of the random finite difference scheme has been studied. Sufficient conditions for the mean square stability of
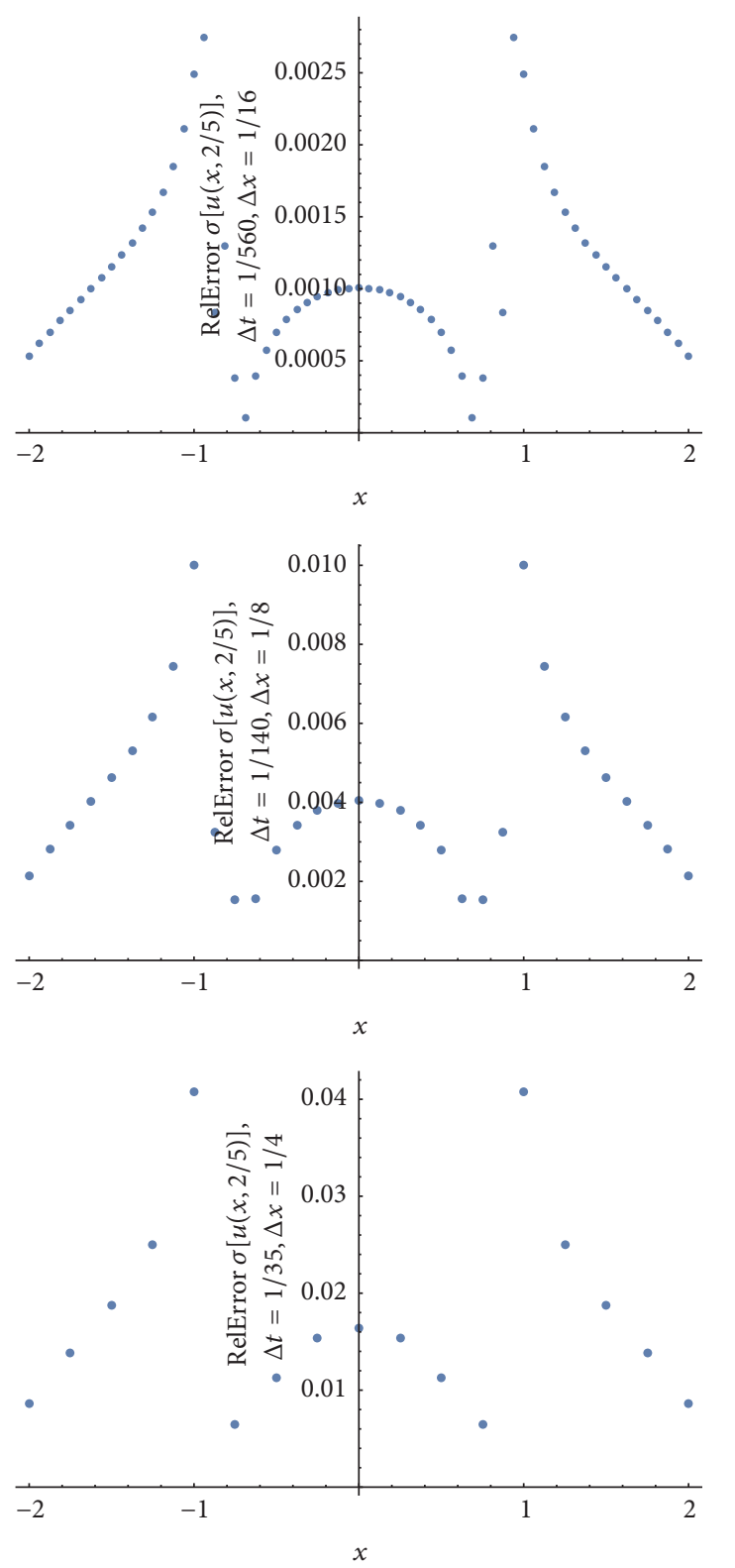

Figure 4: Relative errors at the time instant $t=2 / 5$ for the approximations of the standard deviation for different values of $\Delta x$ and $\Delta t$ over the spatial domain $-2 \leq x \leq 2$.

the random finite difference scheme have been provided. The numerical experiments show that the proposed random finite difference scheme gives reliable approximations for the mean and the standard deviation of the solution stochastic process.

\section{Competing Interests}

The authors declare that there is no conflict of interests regarding the publication of this article.

\section{Acknowledgments}

This work has been partially supported by the Ministerio de Economía y Competitividad Grant MTM2013-41765-P. 
Ana Navarro Quiles acknowledges the doctorate scholarship granted by Programa de Ayudas de Investigación y Desarrollo (PAID), Universitat Politècnica de València. M. A. Sohaly is also indebted to Egypt Ministry of Higher Education, Cultural Affairs, for its financial support [mohe-casem (2016)].

\section{References}

[1] J. H. Lienhard IV and J. H. Lienhard V, A Heat Transfer Textbook, Dover, Mineola, NY, USA, 2013.

[2] F. Kreith, R. M. Manglik, and M. S. Bohn, Principles of Heat Transfer, Cengage Learning, Stamford, Conn, USA, 2011.

[3] J. D. Logan, "Partial differential equations on bounded domains," in Applied Partial Differential Equations, pp. 121-171, Springer, 2004.

[4] M. Kaviany, Heat Transfer Physics, Cambridge University Press, New York, NY, USA, 2014.

[5] D. W. Trim, Applied Partial Differential Equations, PWS-Kent, Boston, Mass, USA, 1990.

[6] L. Wang, X. Zhou, and X. Wei, Heat Conduction: Mathematical Models and Analytical Solutions, Springer, Berlin, Germany, 2008.

[7] Y. A. Çengel and M. A. Boles, Thermodynamics: An Engineering Approach, McGraw-Hill Education, New York, NY, USA, 8th edition, 2015.

[8] J. Wang, "A model of competitive stock trading volume," Journal of Political Economy, vol. 102, no. 1, pp. 127-168, 1994.

[9] D. Z. Wang, "The changes of fishes fauna and protections of aboriginal fishes in the Tarim river," Arid Zone Research, vol. 12, no. 3, pp. 54-59, 1995.

[10] Y. L. Wang, "Prospects of extension for propagation techniques for green cuttages in jujube," Economic Forest Researches, vol. 9, pp. 59-60, 2001.

[11] D. Givoli, Numerical Methods for Problems in Infinite Domains, vol. 33 of Studies in Applied Mechanics, Elsevier, Amsterdam, The Netherlands, 1992.

[12] S. V. Tsynkov, "Numerical solution of problems on unbounded domains. A review," Applied Numerical Mathematics, vol. 27, no. 4, pp. 465-532, 1998.

[13] M. N. Koleva, "Numerical solution of the heat equation in unbounded domains using quasi-uniform grids," in Large-Scale Scientific Computing: 5th International Conference, LSSC 2005, Sozopol, Bulgaria, June 6-10, 2005. Revised Papers, vol. 3743 of Lecture Notes in Computer Science, pp. 509-517, Springer, Berlin, Germany, 2006.

[14] H. Han and Z. Huang, "A class of artificial boundary conditions for heat equation in unbounded domains," Computers and Mathematics with Applications, vol. 43, no. 6-7, pp. 889-900, 2002.

[15] X. Wu and Z.-Z. Sun, "Convergence of difference scheme for heat equation in unbounded domains using artificial boundary conditions," Applied Numerical Mathematics, vol. 50, no. 2, pp. 261-277, 2004.

[16] J. C. Cortés, P. Sevilla-Peris, and L. Jódar, "Analytic-numerical approximating processes of diffusion equation with data uncertainty," Computers and Mathematics with Applications, vol. 49, no. 7-8, pp. 1255-1266, 2005.

[17] M.-C. Casabán, J.-C. Cortés, B. García-Mora, and L. Jódar, "Analytic-numerical solution of random boundary value heat problems in a semi-infinite bar," Abstract and Applied Analysis, vol. 2013, Article ID 676372, 9 pages, 2013.
[18] M.-C. Casabán, R. Company, J.-C. Cortés, and L. Jódar, “Solving the random diffusion model in an infinite medium: a mean square approach," Applied Mathematical Modelling, vol. 38, no. 24, pp. 5922-5933, 2014.

[19] T. T. Soong, Random Differential Equations in Science and Engineering, Academic Press, New York, NY, USA, 1973.

[20] L. Villafuerte, C. A. Braumann, J.-C. Cortés, and L. Jódar, "Random differential operational calculus: theory and applications," Computers and Mathematics with Applications, vol. 59, no. 1, pp. 115-125, 2010.

[21] B. Øksendal, Stochastic Differential Equations: An Introduction with Applications, Springer, Berlin, Germany, 2003.

[22] P. E. Kloeden and E. Platen, Numerical Solution of Stochastic Differential Equations, vol. 23 of Applications of Mathematics, Springer, Berlin, Germany, 1992.

[23] H. Holden, B. Øksendal, J. Ubøe, and T. Zhang, Stochastic Partial Differential Equations: A Modeling, White Noise Functional Approach, Universitext, Springer, New York, NY, USA, 2nd edition, 2010.

[24] E. M. Stein and R. Shakarchi, Functional Analysis: Introduction to Further Topics in Analysis, Princeton Lectures in Analysis, Princeton University Press, Princeton, NJ, USA, 2011. 


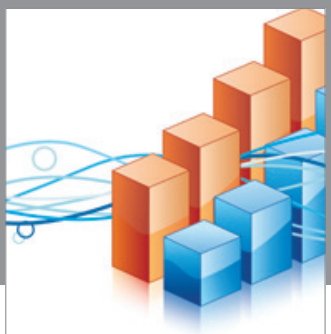

Advances in

Operations Research

vatem alat4

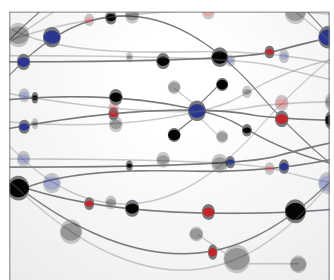

\section{The Scientific} World Journal
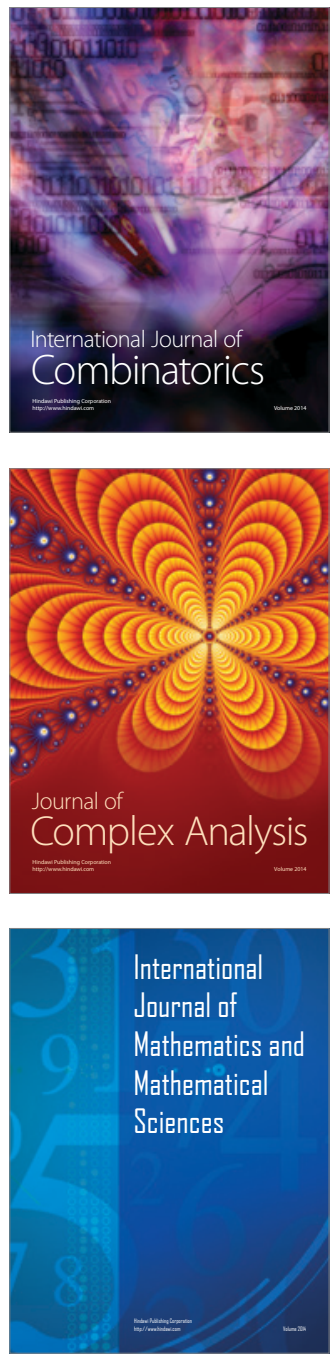
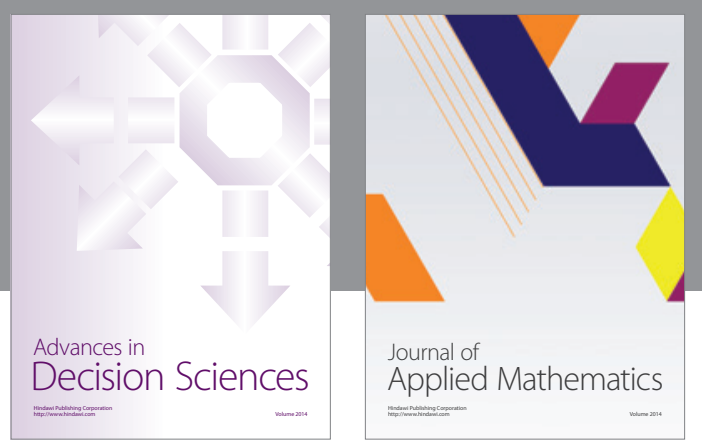

Algebra

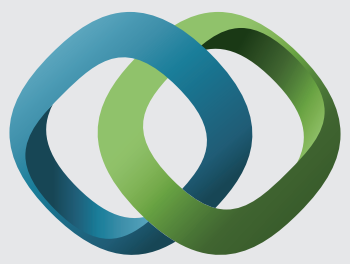

\section{Hindawi}

Submit your manuscripts at

http://www.hindawi.com
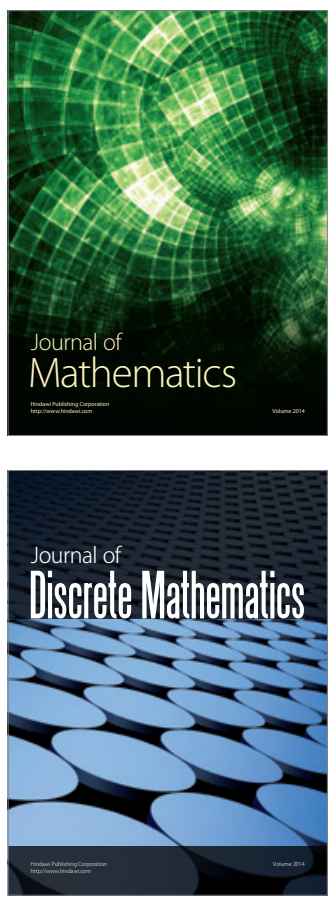

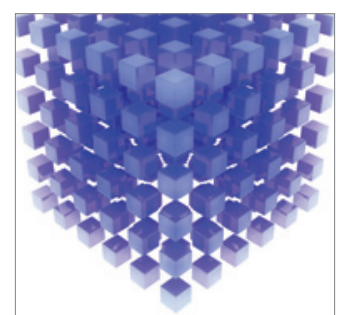

Mathematical Problems in Engineering
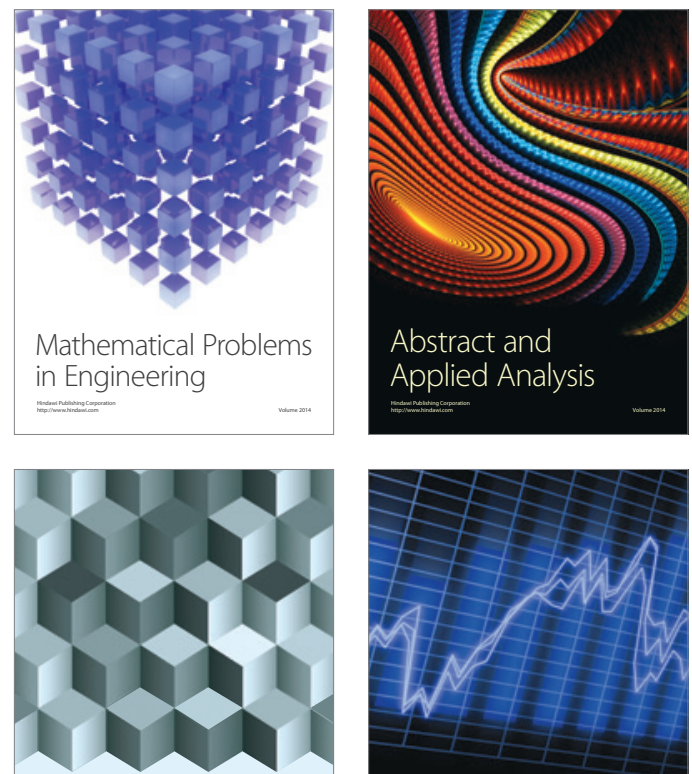

Journal of

Function Spaces

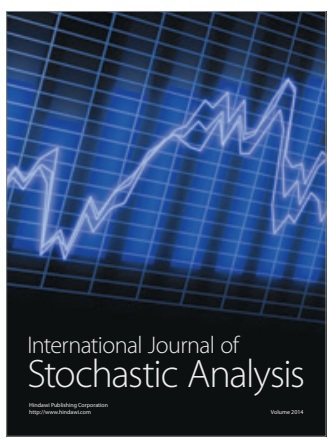

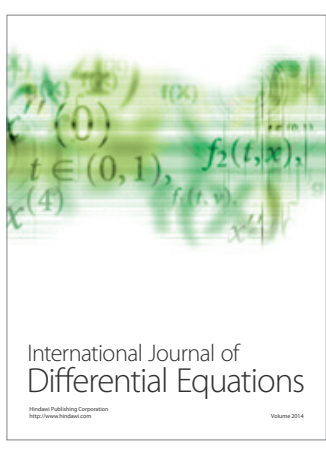
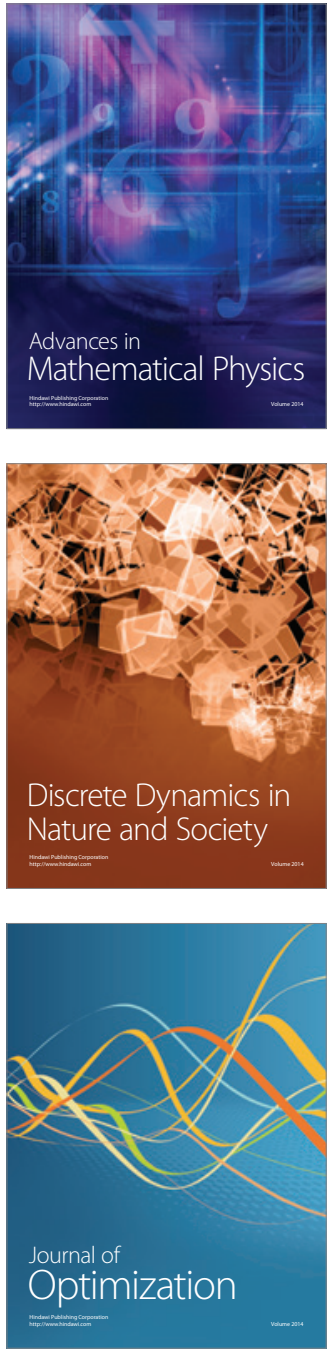